\title{
Herausforderungen für eine zukunftsfähige Strukturpolitik aus gewerkschaftlicher Sicht
}

\section{EINLEITUNG}

Wie eine Mitteilung der EU-Kommission an das Europäische Parlament, den Rat und andere europäische Institutionen zeigt, hat sich die Kommission im November letzten Jahres mit einem der wichtigsten europäischen Vorhaben der nächsten Jahre beschäftigt. Es geht um den wirtschaftlichen, sozialen und territorialen Zusammenhalt Europas, oder anders ausgedrückt: um die Zukunft der Kohäsionspolitik. ${ }^{1}$ In diesem Zusammenhang fordert die Kommission eine strategische Ausrichtung der Strukturfonds auf die EU-2020-Strategie. ${ }^{2}$ Die Kohäsionspolitik soll eine entscheidende Rolle bei der Verwirklichung eines intelligenten, nachhaltigen und integrativen Wachstums spielen.

Der Deutsche Gewerkschaftsbund (DGB) und seine Mitgliedsgewerkschaften sehen die intendierte strategische Ausrichtung der Kohäsionspolitik auf die EU-2020-Strategie kritisch. Für die Gewerkschaften ist die Strukturpolitik ein wichtiges Handlungsfeld der Industriepolitik sowie der Arbeitsmarkt- und Beschäftigungspolitik. Daher hat der DGB sich bereits in den vergangenen Förderperioden frühzeitig in die Diskussion um die $\mathrm{Zu}$ kunft der Strukturfonds eingebracht. Eine ausführliche Stellungnahme zur Zukunft der Kohäsionspolitik wurde im Rahmen des Konsultationsverfahrens im Januar 2011 bei der Kommission eingereicht. ${ }^{3}$

Seitens der Kommission wird die Kohäsionspolitik als Bestandteil einer europäischen Industriepolitik betrachtet: „Eine integrierte Industriepolitik für das Zeitalter der Globalisierung " lautet der Titel der Mitteilung, die eine Diskussion in Gang gebracht hat, welche in Deutschland auf nationaler Ebene aufgegriffen wurde. Der DGB begrüßt dieses politische Signal, das die Rolle der Industriepolitik aufwertet und Wege für die Herausforderungen der nächsten Jahre aufzeigt.

Sowohl die von der EU-Kommission geforderte Ausrichtung der Kohäsionspolitik auf die EU-2020-Strategie als auch die Einbindung in die industriepolitischen Vorhaben erfordern jedoch zunächst eine grundsätzliche Auseinandersetzung mit beiden politischen Zielsetzungen. Der Blick in die Zukunft setzt eine Bilanz der letzten Jahre voraus, um die Fehler, die zur Wirtschafts- und Finanzkrise geführt haben, nicht zu wiederholen.Wie einerseits der sozial-ökologische Wandel bewältigt werden kann und andererseits die Beschäftigten die hierfür notwendigen Qualifikationen erhalten, ist für die Gewerkschaften eine entscheidende Frage, welche es in der Debatte um die Zukunft der Kohäsionspolitik zu beantworten gilt.

Damit dies gelingen kann, liegt ein Fokus dieses Beitrags auf den Bedingungen für eine verbesserte Beteiligung der Wirtschafts- und Sozialpartner (WiSo-Partner) in den Begleitgremien für die Strukturfonds. Sowohl aus Sicht des DGB als auch des Europäischen Gewerkschaftsbundes ist die Qualität der partnerschaftlichen Beteiligung zentrale Voraussetzung für das Gelingen einer nachhaltigen Strukturpolitik in den Mitgliedstaaten und Regionen, denn das Wissen der Akteure vor Ort ist durch nichts zu ersetzen.

\section{DIE STRATEGISCHE AUSRICHTUNG - MAKROÖKONOMISCHE ANFORDERUNGEN}

Der DGB setzt sich ein für ein qualitatives, also soziales, nachhaltiges und ökologisches Wachstum. Das aber heißt zugleich, dass seitens der Gewerkschaften eine Strategie abgelehnt wird, die das Zurückdrängen des Staates fordert, den Glauben an den Markt voraussetzt und auf Fantasie- renditen baut, statt die Realwirtschaft in den Mittelpunkt zu stellen. Ein „weiter so“ kann und darf es nicht länger geben. Dies gilt für die Finanzmärkte, aber auch für die bisher fehlende Koordinierung einer gemeinsamen Wirtschaftspolitik.

Die Lissabonstrategie ist aus Sicht des DGB gescheitert. Sie krankte an der reinen Ausrichtung auf quantitatives Wachstum, was von den Gewerkschaften bereits seit Langem kritisiert wurde. Die in der Lissabonstrategie festgelegten Zielsetzungen einer ökologischen und sozialen Erneuerung wurden gegen ökonomische Ziele ausgespielt. Das darf sich in der EU2020-Strategie nicht wiederholen. Die Argumente hat der DGB im Einzelnen in

1 Vgl. Mitteilung der Kommission an das Europäische Parlament, den Rat, den Europäischen Wirtschafts- und Sozialausschuss, den Ausschuss der Regionen und die Europäische Investitionsbank: Schlussfolgerungen aus dem Fünften Bericht über den wirtschaftlichen, sozialen und territorialen Zusammenhalt: Die Zukunft der Kohäsionspolitik, ec.europa.eu/regional_policy/sources/.../ pdf/conclu_5cr_part1_de.pdf.

2 Vgl. hierzu: Europa 2010: Eine Strategie für intelligentes, nachhaltiges und integratives Wachstum, KOM (2010) 2020 vom 3.3.2010, http:// ec.europa.eu/eu2020/pdf/COMPLET\%20 \%20 DE\%20SG-2010-80021-06-00-DE-TRA-00 pdf.

3 Stellungnahme des DGB im Rahmen der Konsultation der EU-Kommission über die Mitteilung "Schlussfolgerungen aus dem 5. Bericht über den wirtschaftlichen, sozialen und territorialen Zusammenhalt: Die Zukunft der Kohäsionspolitik" vom 31.01.2011, http://ec.europa.eu/regional_ policy/consultation/5cr/pdf/answers/ecosoc/ deutscher_gewerkschaftsbund_bundesvorstand 2011_01_31.pdf.

Christel Degen, Dr., Referatsleiterin im Bundesvorstand des Deutschen Gewerkschaftsbundes (DGB). Arbeitsschwerpunkte: Struktur-, Regionalund Industriepolitik, Aufbau Ost. e-mail: christel.degen@dgb.de 
seiner Stellungnahme „Zur Konsultation der EU-Kommission über die künftige EUStrategie bis $2020^{\text {“ } 4}$ dargelegt. Hier führe ich nur einzelne Beispiele an, die anschaulich machen, warum der DGB dem Ziel der Kommission kritisch gegenübersteht, die Kohäsionspolitik auf die EU-2020-Strategie auszurichten:

(1) Die einseitig auf Wettbewerb ausgerichtete Lissabonstrategie, die damit verbundenen Deregulierungen, Liberalisierungen wie auch eine den Rückzug des Staates propagierende Ideologie konnten - das hat uns die Erfahrung gelehrt - die Wirtschaftskrise nicht verhindern. Das 3-\%-Wachstum wurde - übrigens bereits vor der Wirtschafts- und Finanzkrise nicht erreicht. Konzepte wie die Deregulierung des Arbeitsmarktes haben nichts zu einem „guten Leben“ beigetragen. In den letzen Jahren wurde einmal mehr deutlich, dass Wirtschaftswachstum nicht mit sozialem Fortschritt gleichzusetzen ist.

(2) Der Stabilitäts- und Wachstumspakt muss daher mit qualitativ definierten Zielen der EU-2020-Strategie verknüpft werden, um sicherzustellen, dass der Pakt Investitionen nicht von vornherein verhindert. $\mathrm{Zu}$ den qualitativen Kriterien gehört die umfassende Entwicklung von Indikatoren zur Messung des Kernziels „Nachhaltigkeit“. Weiterhin muss eine Stärkung der Nachfrageseite erfolgen. Zukunftsinvestitionen in Bildung, Forschung und Entwicklung dürfen nicht der Haushaltskonsolidierung zum Opfer fallen. Die Konsolidierung der Haushalte sollte erst dann erfolgen, wenn der Aufschwung selbsttragend ist. Andernfalls droht die permanente Sozialisierung der Verluste durch Krisen.

(3) Die neue Architektur für die Kohäsionspolitik sieht bei Verstößen gegen den Stabilitäts- und Wachstumspakt eine Ausweitung der Sanktionen vor. Selbstredend ist der Einsatz von Fördermitteln ertragreicher, wenn sich das wirtschaftspolitische Umfeld insgesamt positiv entwickelt. Dennoch spricht sich der DGB gegen diesen Ansatz aus, weil das Einfrieren der Fördermittel - und das würde im Sanktionsfall geschehen - für die weitere wirtschaftliche Entwicklung des jeweiligen Landes kontraproduktiv wäre. Auch könnte die Sanktionsdrohung dazu führen, dass strukturpolitische Investitionen von vornherein verhindert werden, da deren Träger unter ggf. unsicheren Finanzierungsmodalitäten agieren müssten. Die Kommission hat auf dieses Problem keine Antwort.

(4) Des Weiteren fordern der DGB und seine Gewerkschaften eine Abkehr vom steuerlichen Unterbietungswettlauf und die Einführung einer europäischen Finanztransaktionssteuer sowie die Wiederherstellung eines vernünftigen Verhältnisses zwischen Finanzwirtschaft und Realwirtschaft: Die angestrebte Konsolidierung der Haushalte bedarf aus gewerkschaftlicher Sicht einer genauen Überprüfung. Dies gilt insbesondere, weil die bisherigen Rettungsmaßnahmen vor allem zulasten der Arbeitnehmerinnen und Arbeitnehmer und des Staates gingen, anstatt - wie es unter dem Gesichtspunkt sozialer Gerechtigkeit geboten wäre - die Verursacher der Finanz- und Wirtschaftskrise bei der Bewältigung und Finanzierung ihrer Folgen mit heranzuziehen.

(5) Die bisher noch unzureichend konkretisierten Vorschläge der EU-Kommission zur „Konditionalität“ betrachten wir mit großer Skepsis: Mit ihrem Vorschlag, die Auszahlung eines Anteils der Strukturfondsmittel von bestimmten wirtschaftlichen und institutionellen Reformen in den Mitgliedstaaten abhängig zu machen, läuft die Kommission Gefahr, die Strukturfonds für die Durchsetzung ihrer - aus unserer Sicht verfehlten - Politik zu instrumentalisieren und das Ziel der Verringerung regionaler Disparitäten innerhalb der EU zu schwächen. Der Vorschlag der SPDBundestagsfraktion, positive Anreize zu setzen und dazu Mittel zu verwenden, die nicht abgerufen worden sind, ist dagegen zu begrüßen.

\section{KOHÄSIONSPOLITIK ALS TEIL DER EUROPÄISCHEN INDUSTRIEPOLITIK}

Nach den Vorstellungen der Kommission ist es die Hauptaufgabe einer europäischen Industriepolitik, optimale Rahmenbedingungen für eine starke, wettbewerbsfähige und diversifizierte industrielle Basis in Europa zu schaffen. Ein Gesamtkonzept zur europäischen Industriepolitik steht jedoch noch aus: Die Mitteilung der Kommission enthält zwar eine Reihe von angekündigten Vorhaben, allerdings ist aus heutiger Sicht noch nicht klar erkennbar, wohin die Reise gehen soll.
Sowohl die industriepolitische Mitteilung als auch die Schlussfolgerungen des 5. Kohäsionsberichts weisen zwar eine gewisse Nachdenklichkeit über die Ursachen und Folgen der Wirtschafts- und Finanzkrise auf, Konsequenzen für die eigene Politik werden jedoch weitgehend vermieden. In beiden Mitteilungen werden letztlich die Leitgedanken der Lissabonstrategie fortgeführt. So soll über die Förderung von Wettbewerbs- und Innovationsfähigkeit die europäische Industrie gestärkt und industrielle Beschäftigung gesichert bzw. geschaffen werden. Jedoch leidet die Industrie in Europa nicht an einer zu geringen Wettbewerbsfähigkeit, sondern es besteht nach wie vor die Gefahr, dass industrielle Kerne dauerhaft verloren gehen und ganze Branchen und Wertschöpfungsketten zusammenbrechen.

Der DGB begrüßt, dass die Kommission in jüngster Zeit verstärkt auf die WiSo-Partner setzt und die positive Rolle der Sozialpartnerschaft in Deutschland bei der Bewältigung der Krise hervorhebt. Dies gilt auch für weitere Initiativen zur Bewältigung des Strukturwandels. Aber die WiSo-Partner benötigen institutionelle und ordnungspolitische Rahmenbedingungen, die ihre Handlungsmöglichkeiten unterstützen und zur Entfaltung bringen. Die europäische Industriepolitik soll für die europäischen Unternehmen einen fairen Wettbewerb mit gleichen Spielregeln schaffen. Ein aktiver Staat muss den stattfindenden Strukturwandel an traditionellen Industriestandorten nachhaltig, das heißt ökonomisch erfolgreich, sozial und ökologisch ausgerichtet, gestalten. Dazu ist eine grundsätzliche Abkehr vom bisherigen Ansatz nötig, der davon ausgeht, dass die Stärkung der Wettbewerbsfähigkeit von Unternehmen in Selbstregulation mehr und bessere Arbeitsplätze schaffen kann.

Rückblickend hat die Weltwirtschaftskrise in Deutschland und weit darüber hinaus dafür gesorgt, dass von einer Renaissance der Industriepolitik die Rede ist. DGB und Gewerkschaften betrachten die Industriepolitik nicht nur als kurzfristiges Instrument zur Arbeitsplatzsicherung und zum Unternehmenserhalt in der Krise:

4 Stellungnahme des DGB zur Konsultation der EU-Kommission über die künftige EU-Strategie bis 2020 vom 18.1.2010, www.einblick.dgb.de/ hintergrund/2010/03/dgb_stellunnahme_eu. pdf/. 
Eine gezielte Beeinflussung des Strukturwandels durch industrie- und strukturpolitische Interventionen muss aus gewerkschaftlicher Sicht jetzt die Weichen für zukunftsfähige Strukturen stellen.

Fundamental ist für den DGB ein neues Anreizsystem auf den Kapitalmärkten. Dieses sollte langfristige Realinvestitionen fördern und kurzfristige Spekulationen diskriminieren. $\mathrm{Zu}$ den wichtigsten makroökonomischen Aufgaben gehört ein Nachhaltigkeitscheck der bisherigen Finanzmarktliberalisierung. Wo offensichtliche Fehlanreize gesetzt wurden, gilt es umzusteuern. Zentrales Kriterium ist die Frage, ob die aktuellen Regeln eine ökonomisch, sozial und ökologisch nachhaltige Unternehmenspolitik fördern oder nicht.

Die EU-2020-Strategie betont zwar den ökologischen Umbau der Industrie, jedoch gehen die avisierten Ziele (Stichwort „20-20-20“) nicht weit genug. Der DGB spricht sich für eine „grüne“ Industrierevolution aus, die eine drastische Umsteuerung notwendig macht. Innovation, grüne Förderpolitik und ein neues (Umwelt-) Bewusstsein sind hierfür nötig. Die Unternehmen in der Umweltindustrie müssen massiv gefördert werden, damit die EU (Deutschland: $16 \%$ Weltmarktanteil) wettbewerbsfähig bleibt und den ökologischen Know-how-Vorsprung künftig nicht einbüßt.

\section{DEN STRUKTURWANDEL GESTALTEN - GUTE ARBEIT SCHAFFEN}

Die Strukturfonds sind ein zentrales Instrument, um den strukturellen Wandel in Europa zu bewältigen, regionale Disparitäten zu verringern und ein soziales Europa zu schaffen. Die Kommission betont, dass die Beteiligung der Sozialpartner erhöht und die partnerschaftliche Beteiligung insgesamt verbessert werden soll. Die Europäische Union steht vor vielen Herausforderungen. Dazu gehören der verstärkte wirtschaftliche Druck der globalen Konkurrenz, der für 2020 erwartete - und in vielen Regionen bereits stattfindende - Rückgang der Bevölkerungszahlen wie auch der fortschreitende Alterungsprozess, steigende Energiepreise sowie Klimawandel und soziale Polarisierung.

Wie können Regionen auf den Restrukturierungsdruck dynamischer Wettbewerber im Low- und Medium-Tech-Bereich reagieren? Wie kann der innere Zusammenhalt in Europa unter diesen Bedin- gungen ein integriertes Konzept für Entwicklung, qualitatives Wachstum und Arbeitsplätze erarbeiten? Und wie kann diese Politik der Unterschiedlichkeit der EU-Regionen Rechnung tragen? ${ }^{5}$ Das sind nur einige der Schlüsselfragen, auf die Europa Antworten finden muss.

Bei der Integration der zehn neuen Mitgliedsländer in der laufenden Förderperiode (2007-2013) spielen die Strukturfonds eine wichtige Rolle. Aus den Mitteln des Europäischen Sozialfonds (ESF) werden zur Zeit jährlich über $10 \mathrm{Mrd}$. investiert, damit Arbeitnehmerinnen und Arbeitnehmer ihre beruflichen Qualifikationen und damit ihre Vermittelbarkeit verbessern können.

Deutschland wird laut den Angaben des Bundesministeriums für Wirtschaft in der laufenden Förderperiode 2007-2013 EU-Strukturfondsmittel in Höhe von rund 26,3 Mrd. € (in aktuellen Preisen) erhalten. Regionen, die nach dem Konvergenzziel förderfähig sind, erhalten davon rund 16,1 Mrd. $€$. Dies sind die neuen Bundesländer (Mittelvolumen rund 15,3 Mrd. €). $\mathrm{Zu}$ den Phasing-Out Regionen gehören Brandenburg-Südwest, Halle, Leipzig und Lüneburg.

Die Strukturfonds waren laut Beschluss des Europäischen Rates die notwendigen finanziellen Instrumente zur Umsetzung der Lissabonstrategie, wobei die beruflich am geringsten qualifizierten Menschen und diejenigen, die beim Zugang zur aktiven nationalen Beschäftigungspolitik die größten Schwierigkeiten zu überwinden haben, im Mittelpunkt stehen sollten. ${ }^{6}$ In diesem Zusammenhang ist der ESF das vorrangige Instrument zur Umsetzung der europäischen Beschäftigungsleitlinien und muss es auch zukünftig im Rahmen der EU-2020-Strategie bleiben.

Ebenso muss der wirtschaftliche, soziale und territoriale Zusammenhalt weiterhin im Zentrum der EU-2020-Strategie stehen, um sicherzustellen, dass alle Fähigkeiten und Kräfte mobilisiert und für die Umsetzung der Strategie eingesetzt werden. Die Strukturfonds sind die zentralen Instrumente für die Verwirklichung der als „intelligentes, nachhaltiges und integratives Wachstum" bezeichneten Priorität in den Mitgliedstaaten, Regionen und Gebieten.

Die Bekämpfung sozialer Ungleichheit und Armut ist ein wichtiges Ziel der EU-2020-Strategie. Eine Stärkung der Binnennachfrage zur Stabilisierung der Wirtschaft und zur Verringerung der Einkom- mensungleichheiten sowie die Integration Jugendlicher müssen weitere vorrangige Ziele sein.

Die Qualität der Arbeit ist dabei entscheidend: „Gute Arbeit“ umfasst sozialen Schutz, faire Löhne, Arbeitsplatzsicherheit, Gesundheitsschutz sowie eine familienfreundliche Arbeitsorganisation. Die EU-Kommission muss dafür messbare Qualitätskriterien einführen. Das setzt u. a. voraus, die wöchentlich geleisteten Arbeitsstunden zu erfassen, gleiche Löhne für Frauen und Männer durchzusetzen, gesetzliche, tarifliche Regelungen und Vereinbarungen zu Mindestlöhnen sowie die soziale Sicherung einzuhalten.

Die wichtigste Herausforderung für eine zukunftsfähige Strukturpolitik besteht aus unserer Sicht darin, den Strukturwandel zu bewältigen. „Grüne Jobs“ können in fast allen Industrie- und Dienstleistungssektoren entstehen. Investitionen in den ökologischen Wandel würden viele neue Arbeitsplätze und neue Einkommensmöglichkeiten schaffen. Eine solche Strategie begrüßt der DGB. Er mahnt allerdings zugleich an, den großen Anpassungsbedarf, den der Wandel mit sich bringt, aktiv im Sinne der Beschäftigten zu gestalten. Die EU-2020 Strategie erwähnt demgegenüber diese Herausforderung mit keinem Wort.

„Green Jobs“ gehen nicht automatisch mit guten Arbeitsbedingungen einher, wie die Erfahrungen aus der Solarindustrie in Ostdeutschland zeigen. Die EU-2020-Strategie und die Strukturfonds müssen daher gute Arbeitsbedingungen fördern. Initiativen der Kommission, den anstehenden Strukturwandel gemeinsam mit den WiSo-Partnern zu antizipieren („Anticipating and managing restructuring in a socially responsible way - New partnerships to preserve employment ${ }^{\text {“7 }}$ oder „New Skills

5 Vgl. Stellungnahme der Arbeitsgemeinschaft Europäischer Grenzregionen (AGEG) „Wachsende Regionen, wachsendes Europa - Konsultationen der Öffentlichkeit zur Zukunft der europäischen Kohäsionspolitik" vom 29.01.2008, http://www. aebr.eu//files/filemanager_files//test/News/ ARFE_Position_5_me_Rapport_de_Coh_sion_ FR.pdf.

6 Vgl. Stellungnahme des EWSA vom 12. Juli 2010 zum Thema Beschäftigung für vorrangige Bevölkerungsgruppen (Lissabon Strategie) (SOC/251), Berichterstatter: Wolfgang Greif (Abl. C 256, 27.10.2007).

7 Report on 27 National Seminars on Anticipating and Managing Restructuring - A.R.E.NA.S (Service contract VC/ 2008/0667), EU Synthesis report (27), September 2010 
for New Jobs (") ) weisen grundsätzlich in die richtige Richtung. Hier kommt es jedoch darauf an, dass Europa sich von Niedriglohnstrategien, die unter dem Deckmantel der Flexibilität offenbar neu belebt werden sollen, verabschiedet.

Deshalb fordert der DGB die Orientierung von Forschung und Entwicklung an Kriterien der Nachhaltigkeit, die Weiterentwicklung verbindlicher Energieeffizienzstandards sowie die Einleitung von Ausbildungsinitiativen im Bereich Umwelttechnologie - Förderung von Aus- und Weiterbildung, um neue Kompetenzen zu schaffen, die für "Green Jobs“ gebraucht werden.

Der DGB unterstützt die Auffassung des Europäischen Parlaments (EP), dass eine Verwirklichung der Ziele der Strategie 2020 voraussetzt, besonderes Gewicht auf die Modernisierung der Bildungssysteme und der Berufsbildungs- und Schulungsmaßnahmen zu legen. Weitere Ziele des EPs sind die Förderung menschenwürdiger Arbeit durch Bekämpfung prekärer Beschäftigungsverhältnisse, die Gleichstellung der Geschlechter, die Vereinbarkeit von Erwerbs- und Privatleben sowie die Möglichkeit zur demokratischen Teilhabe. Für das EP muss außerdem gewährleistet sein, dass derzeit vom Arbeitsmarkt ausgeschlossene Menschen zu diesem Zugang bekommen." ${ }^{\text {"9 }}$

\section{PARTNERSCHAFT BRAUCHT ECHTE TEILHABE}

Das Partnerschaftsprinzip, das sowohl für die Sozialpartner als auch für andere Organisationen der organisierten Zivilgesellschaft gilt, ${ }^{10}$ ist der wesentliche Garant für ein gutes Funktionieren der Maßnahmen im Rahmen der Strukturfonds. Eine Partnerschaft auf Augenhöhe - so die Erfahrung des europäischen Wirtschafts- und Sozialausschusses (EWSA) - verbessert den Erfolg der Kohäsionspolitik deutlich. Dies haben Erfahrungen und Evaluationen gezeigt. Die gelebte Umsetzung des Partnerschaftsprinzips ist ein Instrument nachhaltiger Entwicklung. Sie erhöht die Sichtbarkeit der EU und stärkt die Demokratie. Die geltenden Verordnungen lassen jedoch unterschiedlichen Interpretationen des Partnerschaftskonzeptes noch zu viel Raum. Deshalb fordert der EWSA eine aktivere Rolle der Kommission bei der Verbreitung dieses Prinzips. Die Verordnungen müssen beim Partnerschaftsprinzip angeglichen werden, um das Engagement der Sozialpartner in allen Fonds zu gewährleisten.

Der EWSA hat in seiner Stellungnahme „Förderung einer effizienten Partnerschaft bei der Verwaltung des neuen Programmplanungszeitraums in der Kohäsionspolitik 2007-2013 unter Rückgriff auf bewährte Verfahrensweisen“ (Berichterstatter: Jan Olssen) vom Juni 2010 ausgeführt, welche Faktoren zu einer Verbesserung des Partnerschaftsprinzips beitragen können. ${ }^{11}$ Die frühzeitige, verpflichtende Konsultation der Sozialpartner in allen Phasen der ,integrierten Leitlinien für Wachstum und Beschäftigung“ und der „offenen Koordinierung" sowie die frühzeitige, verpflichtende Einbeziehung der Sozialpartner in Evaluationsprozesse und nicht zuletzt die Herstellung von Transparenz über die Verhandlungsentwicklung müssen sichergestellt werden.

Kontinuierlicher Kapazitätsaufbau der Partner ist dabei von entscheidender Bedeutung. Dem ESF kommt eine besondere Aufgabe bei der Stärkung der Sozialpartner und des sozialen Dialogs zu. Der ESF sollte Partnerschaften, Bündnisse und Initiativen durch Vernetzung der relevanten Akteure, zum Beispiel der Sozialpartner und der Nicht-Regierungsorganisationen, auf transnationaler, nationaler, regionaler und lokaler Ebene fördern. Dies sollte durch Kapazitätsstärkung unterstützt werden.

Der EWSA betont, dass es eines Lernprozesses bedarf, um echte Partnerschaft zu erreichen. Dabei geht es um die Befähigung der Partner und die Schaffung gleicher Bedingungen für alle, damit diese in allen Phasen der Durchführung der Kohäsionspolitik teilhaben können. Eine zentrale Forderung ist deshalb, dass Ressourcen für den Kapazitätsaufbau in allen operationellen Programmen und allen Strukturfonds (nicht nur im ESF) genutzt werden können. Dies soll zukünftig in allen Regionen ermöglicht werden.

Mit Blick auf die Erfüllung von Mindestanforderungen, die zu einem hohen Standard bei den Partnerschaften führen, schlägt der EWSA vor, dass die Kommission zusammen mit Vertretern der in Artikel 11 genannten Kategorien von Partnern einen Verhaltenskodex bezüglich der Durchführung der Partnerschaft erarbeitet. Dieser soll auf folgende Leitlinien gestützt sein: ${ }^{12}$

(1) Die entsprechenden Partner sollten anfangs einen Mitwirkungsplan für die Pro- grammplanung, Umsetzung und Bewertung vereinbaren, der klare Zielsetzungen und einen Fahrplan enthält.

(2) Die zuständigen Behörden sollten Rechenschaft ablegen, wie sie in ihren Vorschlägen und Berichten das Partnerschaftsprinzip berücksichtigt haben. Die Ansichten der Partner sollten klar und offen dokumentiert werden.

(3) Den Partnerschaften sollte die Verantwortung für das Verfahren zur Wahl ihrer eigenen Mitglieder in die Begleitausschüsse und weitere beratende Gremien übertragen werden, die im Rahmen der Strukturfonds eingerichtet worden sind. Für die Wahl sollten Interessen, Repräsentativität und Offenheit bei neuen, engagierten und innovativen Akteuren maßgeblich sein.

(4) Die Partnerschaften sollten von Beginn an in die Festlegung von Projektauswahlkriterien einbezogen werden.

(5) Partnerschaft sollte ein Kriterium für von Fonds finanzierte Projekte sein; insbesondere transnationale Partnerschaften sollten gefördert werden.

(6) Es sollten Indikatoren für eine effiziente Verwaltung aus dem Blickwinkel der Begünstigten geschaffen werden.

(7) Verfahren und Kontrollen sollten vereinfacht, Zahlungen an Begünstigte beschleunigt werden.

(8) Langfristige Strategien sollten in den Lenkungsausschüssen oder sonstigen geeigneten Gremien erörtert werden, die für die einzelstaatlichen strategischen Rahmenpläne (ESRP) zuständig sind.

(9) Allen Partnerschaften sollte technische Hilfe in allen operationellen Programmen

8 New Skills for New Jobs: Action Now A report by the Expert Group on New Skills for New Jobs prepared for the European Commission.

9 Vgl. Entschließung des Europäischen Parlaments vom 7 Oktober 2010 zur Zukunft des Europäischen Sozialfonds (Punkt 6.).

10 Wie in Art. 11 der Verordnung (EG) 1083/2006 des Rates vom 11. Juli 2006 mit allgemeinen Bestimmungen über den Europäischen Fonds für regionale Entwicklung, den Europäischen Sozialfonds und den Kohäsionsfonds festgelegt.

11 Die folgenden Ausführungen fassen die wichtigsten Forderungen des Olssen-Papiers zusammen.

12 Vgl. Ebd. 
für den Kapazitätsaufbau, die Koordinierung und Repräsentation zur Verfügung stehen, wobei die Partner selbst solche Projekte ausarbeiten und verwalten.

In Deutschland wurden in den Konvergenzgebieten mit Beratungsstellen, die über die technische Hilfe finanziert wurden, bis heute sehr gute Erfahrungen gemacht. Hier konnte mit vergleichsweise geringen Mitteln die Effizienz erhöht und die Bürokratie verringert werden.

Inzwischen ist es dem DGB in einigen Bundesländern gelungen, Kriterien „Guter Arbeit" als Voraussetzung für die Vergabe von Strukturfondsmitteln in den Begleitausschüssen zu vereinbaren. Dies gilt beispielsweise in Mecklenburg-Vorpommern und Sachsen-Anhalt für die Nichtförderung von Leiharbeit und in Brandenburg,
Sachsen, Sachsen-Anhalt und Thüringen für Mindestentgelte. ${ }^{13}$ Dieser Weg muss weiter verfolgt werden. Die Qualität der Arbeitsverhältnisse muss als Ziel der Wirtschaftsförderung aufgenommen werden.

13 Vgl. Ziegler, A. (2009): Strukturpolitik in der Krise - Mehr Standortwettbewerb und Innovationsförderung, in: WSI-Mitteilungen 62 (5), S. 260-267. 